\title{
Geometries on $\sigma$-Hermitian matrices
}

\author{
Andrea Blunck Hans Havlicek
}

\section{Introduction: Square Matrices}

Ring geometry and the geometry of matrices meet naturally at the $\operatorname{ring} R:=K^{n \times n}$ of $n \times n$ matrices with entries in a (not necessarily commutative) field $K$. Our aim is to strengthen the interaction between these disciplines. Below we sketch some results from either side, even though not in their most general form, but in a way which is tailored for our needs.

Let us start with ring geometry, where we follow [7] and [10]: Consider the free left $R$-module $R^{2}$ and the group $\mathrm{GL}_{2}(R)=\mathrm{GL}_{2 n}(K)$ of invertible $2 \times 2$-matrices with entries in $R$. A pair $(A, B) \in R^{2}$ is called admissible if there exists a matrix in $\mathrm{GL}_{2}(R)$ with $(A, B)$ being its first row. The projective line over $R$, in symbols $\mathbb{P}(R)$, is the set of cyclic submodules $R(A, B)$ for all admissible pairs $(A, B) \in R^{2}$. Two admissible pairs represent the same point precisely when they are left-proportional by a unit in $R$, i. e., a matrix from $\mathrm{GL}_{n}(K)$. Conversely, if $R\left(A^{\prime}, B^{\prime}\right)=R(A, B)$ for some pair $\left(A^{\prime}, B^{\prime}\right) \in R^{2}$ and an admissible pair $(A, B) \in R^{2}$ then $\left(A^{\prime}, B^{\prime}\right)$ is admissible too [3, Proposition 2.2]. By [2], the projective line over $R$ allows the following description which is not available for arbitrary rings, as it makes use of the left row rank of a matrix $X$ over $K$ (in symbols: $\operatorname{rank} X$ ):

$$
\mathbb{P}(R)=\{R(A, B) \mid A, B \in R, \operatorname{rank}(A, B)=n\} .
$$

Here $(A, B) \in R^{2}$ has to be interpreted as the $n \times 2 n$ matrix over $K$ arising from $A$ and $B$ by means of horizontal augmentation. Because of (1), the point set of $\mathbb{P}(R)$ is in bijective correspondence with the Grassmannian $\mathrm{Gr}_{2 n, n}(K)$ comprising all $n$-dimensional subspaces of the left $K$-vector space $K^{2 n}$ via

$$
\mathbb{P}(R) \rightarrow \mathrm{Gr}_{2 n, n}(K): R(A, B) \mapsto \text { left row space of }(A, B) \text {. }
$$

From [13, 2.6], our matrix ring $R=K^{n \times n}$ has stable rank 2 [13, § 2]. Viz. for each $(A, B) \in R^{2}$ which is unimodular, i. e., there are $X, Y \in R$ with $A X+B Y=I$, there exists $W \in R$ such that $A+B W \in \mathrm{GL}_{n}(K)$. Consequently, two important results 
hold: Firstly, any unimodular pair $(A, B) \in R^{2}$ is admissible [13, 2.11]. Secondly, Bartolone's parametrisation

$$
R^{2} \rightarrow \mathbb{P}(R):\left(T_{1}, T_{2}\right) \mapsto R\left(T_{2} T_{1}-I, T_{2}\right)
$$

is well defined and surjective. This allows us to write the projective line $\mathbb{P}(R)$ in the form

$$
\mathbb{P}(R)=\left\{R\left(T_{2} T_{1}-I, T_{2}\right) \mid T_{1}, T_{2} \in R\right\} .
$$

See [1] and compare with [4] for a generalisation.

Let us now switch to the geometry of matrices, where [14] is our standard reference. By comparing the description of the point set $\mathbb{P}\left(K^{n \times n}\right)=\mathbb{P}(R)$ in (1) with the definition of the point set of the projective space of $m \times n$ matrices over $K$ in $[14,3.6]$, one sees immediately that the two definitions coincide for $m=n \geq 2$ up to the immaterial fact that we address a Grassmannian in the vector space $K^{2 n}$ rather than in the projective space on $K^{2 n}$. The bijection from (2) turns (3) into a surjective parametric representation of the Grassmannian $\mathrm{Gr}_{2 n, n}(K)$, namely

$$
R^{2} \rightarrow \operatorname{Gr}_{2 n, n}(K):\left(T_{1}, T_{2}\right) \mapsto \text { left row space of }\left(T_{2} T_{1}-I, T_{2}\right) .
$$

A major difference concerns the additional structure which is imposed on $\mathrm{Gr}_{2 n, n}(K)$ : In the ring-geometric setting the point set $\mathbb{P}(R)$ is endowed with the symmetric and anti-reflexive relation distant $(\triangle)$ defined by

$$
R(A, B) \triangle R(C, D) \quad \Leftrightarrow \quad\left(\begin{array}{ll}
A & B \\
C & D
\end{array}\right) \in \mathrm{GL}_{2}(R) .
$$

Being distant is equivalent to the complementarity of the $n$-dimensional subspaces of $K^{2 n}$ which correspond via (2). A crucial property of the projective line over our $\operatorname{ring} R$, and more generally over any ring of stable rank 2 , is as follows [10, 1.4.2]: Given any two points $p$ and $q$ there exists some point $r$ such that $p \Delta r \Delta q$. In the matrix-geometric setting two $n$-dimensional subspaces of $K^{2 n}$ are called adjacent $(\sim)$ if, and only if, their intersection has dimension $n-1$. However, adjacency can be expressed in terms of being distant and vice versa [5, Theorem 3.2]. See also $[12,3.2 .4]$, where complementary subspaces are called opposite.

We refer to [6] for several applications of this link between $\mathbb{P}(R)$ and the Grassmannian $\mathrm{Gr}_{2 n, n}(K)$, like a unified explicit description of adjacency preserving transformations of $\mathrm{Gr}_{2 n, n}(K)$ which avoids the usual distinction between semilinear bijections and non-degenerate sesquilinear forms.

\section{$2 \sigma$-Hermitian matrices}

Suppose now that the field $K$ admits an involution, i. e. an antiautomorphism $\sigma$ such that $\sigma^{2}=\mathrm{id}_{K}$. As before, we let $R=K^{n \times n}$. The involution $\sigma$ determines an 
antiautomorphism of $R$, namely the $\sigma$-transposition $M=\left(m_{i j}\right) \mapsto\left(M^{\sigma}\right)^{\mathrm{T}}:=\left(m_{j i}^{\sigma}\right)$. The elements of $H_{\sigma}:=\left\{X \in R \mid X=\left(X^{\sigma}\right)^{\mathrm{T}}\right\}$ are the $\sigma$-Hermitian matrices of $R$. (In the special case that $\sigma=\mathrm{id}_{K}$ the field $K$ is commutative, and we obtain the subset of symmetric matrices of $K^{n \times n}$.) The set $H_{\sigma}$ need not be closed under matrix multiplication. In the terminology of [7, 3.1.5], $H_{\sigma}$ is a Jordan system of $R$, where $R=K^{n \times n}$ is considered as an algebra over the centre $Z(K)$ of $K$. This means that $H_{\sigma}$ is a subspace of the $Z(K)$-vector space $R$ which contains $I$, and which has the property that

$$
A^{-1} \in H_{\sigma} \text { for all } A \in \mathrm{GL}_{n}(K) \cap H_{\sigma} .
$$

Moreover, $H_{\sigma}$ is Jordan closed, i. e., it satisfies the condition

$$
A B A \in H_{\sigma} \text { for all } A, B \in H_{\sigma} .
$$

We follow $[7,3.1 .14]$ by defining the projective line $\mathbb{P}\left(H_{\sigma}\right)$ over $H_{\sigma}$ as

$$
\mathbb{P}\left(H_{\sigma}\right)=\left\{R\left(T_{2} T_{1}-I, T_{2}\right) \mid T_{1}, T_{2} \in H_{\sigma}\right\} .
$$

From (4), $\mathbb{P}\left(H_{\sigma}\right)$ is a subset of $\mathbb{P}(R)$. It is important to point out that $\mathbb{P}\left(H_{\sigma}\right)$ is not defined as the set of all $R(A, B)$ with $(A, B)$ admissible and $A, B \in H_{\sigma}$. Nevertheless, all points $R(A, I)$ and $R(I, A)$ with $A \in H_{\sigma}$ belong to $\mathbb{P}\left(H_{\sigma}\right)$.

We now recall the definition of the projective space of $\sigma$-Hermitian matrices from [9, III $\S 3]$ and [14, 6.8]. Let $\beta: K^{2 n} \times K^{2 n} \rightarrow K$ be the non-degenerate $\sigma$-antiHermitian sesquilinear form given by the matrix

$$
\left(\begin{array}{cc}
0 & I_{n} \\
-I_{n} & 0
\end{array}\right) \in \mathrm{GL}_{2 n}(K)
$$

The form $\beta$ is trace-valued and has Witt index $n$. The subset of the Grassmannian $\mathrm{Gr}_{2 n, n}(K)$ comprising all maximal totally isotropic subspaces is the point set of the projective space of $\sigma$-Hermitian matrices or, in another terminology, the point set of the dual polar space given by $\beta$; see [8] or $[12,4.1]$.

Suppose that $(A, B) \in R^{2}$ satisfies $\operatorname{rank}(A, B)=n$. By [14, Proposition 6.41], the (n-dimensional) left row space of $(A, B) \in K^{n \times 2 n}$ is totally isotropic if, and only if,

$$
A\left(B^{\sigma}\right)^{\mathrm{T}}=B\left(A^{\sigma}\right)^{\mathrm{T}} \text {. }
$$

Thus it is easy to decide whether or not an element of the Grassmannian $\mathrm{Gr}_{2 n, n}(K)$ is totally isotropic. For example, all pairs $(A, I)$ and $(I, A)$ with $A \in H_{\sigma}$ give rise to maximal totally isotropic subspaces.

Note that our Jordan system $H_{\sigma}$ need not be strong (in German: "starkes JordanSystem") in the sense of [7, 3.1.5], as we do not assume any richness conditions. Also, we did not adopt the extra assumptions on $\sigma$ from [14, p. 306]. 
By the above, the set of $\sigma$-Hermitian matrices gives rise to two subsets of $\mathrm{Gr}_{2 n, n}(K)$. The coincidence of these two subsets is not obvious. Indeed, in the ring-geometric setting the subset is given in terms of a parametric representation, whereas in the matrix-geometric setting there is a defining matrix equation. Our main result states that the two subsets coincide.

Theorem 1 ([6]). Let $K$ be any field admitting an involution $\sigma$. The point set of the projective space of $\sigma$-Hermitian $n \times n$ matrices over $K$ coincides with the projective line over the Jordan system $H_{\sigma}$ of all $\sigma$-Hermitian matrices of $R=K^{n \times n}$.

Our proof of this theorem uses two auxiliary results about dual polar spaces. The first is rather technical.

Lemma 1 ([6]). Let $U=V \oplus W$ be a maximal totally isotropic subspace of $\left(K^{2 n}, \beta\right)$ which is given as direct sum of subspaces $V$ and $W$. Then there exists a maximal totally isotropic subspace, say $X$, such that $X \cap V^{\perp}=W$.

With this result at hand the following can be established:

Lemma 2 ([6]). Let $U_{1}$ and $U_{2}$ be two maximal totally isotropic subspaces of $\left(K^{2 n}, \beta\right)$. Then there exists a maximal totally isotropic subspace $X$ which is a common complement of $U_{1}$ and $U_{2}$.

Sketch of the proof of Theorem 1 . The proof of one inclusion simply amounts to plugging in representatives of the points from (8) in the matrix equation (10). Conversely, let the left row space of $(A, B)$ be a maximal totally isotropic subspace. By Lemma 2, there exists a maximal totally isotropic subspace of $K^{2 n}$ which is a common complement of the left row spaces of $(I, 0)$ and $(A, B)$. In matrix form it can be written as $(C, I)$ with $C \in H_{\sigma}$. So, in terms of $\mathbb{P}(R)$, we have $R(I, 0) \triangle R(C, I) \triangle R(A, B)$. Defining $T_{1}:=C$ and $T_{2}:=(B C-A)^{-1} B$ gives after some calculations that $R(A, B)=R\left(T_{2} T_{1}-I, T_{2}\right)$ and $R(A, B) \in \mathbb{P}\left(H_{\sigma}\right)$.

In view of Theorem 1 one may carry over results from $\mathbb{P}\left(H_{\sigma}\right)$ to the projective space of $\sigma$-Hermitian matrices; see [6].

Finally, let us mention two open problems:

1. Is it possible to express the adjacency relation on a projective space of $\sigma$ Hermitian matrices in terms of the distant relation on $\mathbb{P}\left(H_{\sigma}\right)$ ?

See [6], [11] and [12, 4.7.1] for further details.

2. Is it possible to extend the present results from the matrix ring $R=K^{n \times n}$ to other rings which admit an anti-automorphism?

An affirmative answer would give, mutatis mutandis, an alternative approach to projective lines over certain Jordan systems in terms of a defining equation similar to (10). 


\section{References}

[1] C. Bartolone. Jordan homomorphisms, chain geometries and the fundamental theorem. Abh. Math. Sem. Univ. Hamburg, 59:93-99, 1989.

[2] A. Blunck. Regular spreads and chain geometries. Bull. Belg. Math. Soc. Simon Stevin, 6:589-603, 1999.

[3] A. Blunck and H. Havlicek. Projective representations I. Projective lines over rings. Abh. Math. Sem. Univ. Hamburg, 70:287-299, 2000.

[4] A. Blunck and H. Havlicek. Jordan homomorphisms and harmonic mappings. Monatsh. Math., 139:111-127, 2003.

[5] A. Blunck and H. Havlicek. On bijections that preserve complementarity of subspaces. Discrete Math., 301:46-56, 2005.

[6] A. Blunck and H. Havlicek. Projective lines over Jordan systems and geometry of Hermitian matrices. Linear Algebra Appl., 433:672-680, 2010.

[7] A. Blunck and A. Herzer. Kettengeometrien - Eine Einführung. Shaker Verlag, Aachen, 2005.

[8] P. J. Cameron. Dual polar spaces. Geom. Dedicata, 12(1):75-85, 1982.

[9] J. A. Dieudonné. La Géométrie des Groupes Classiques. Springer, Berlin Heidelberg New York, 3rd edition, 1971.

[10] A. Herzer. Chain geometries. In F. Buekenhout, editor, Handbook of Incidence Geometry, pages 781-842. Elsevier, Amsterdam, 1995.

[11] M. Kwiatkowski and M. Pankov. Opposite relation on dual polar spaces and halfspin Grassmann spaces. Results Math., 54(3-4):301-308, 2009.

[12] M. Pankov. Grassmannians of Classical Buildings, volume 2 of Algebra and Discrete Mathematics. World Scientific, Singapore, 2010.

[13] F. D. Veldkamp. Projective ring planes and their homomorphisms. In R. Kaya, P. Plaumann, and K. Strambach, editors, Rings and Geometry, pages 289-350. D. Reidel, Dordrecht, 1985.

[14] Z.-X. Wan. Geometry of Matrices. World Scientific, Singapore, 1996.

Andrea Blunck, Department Mathematik, Universität Hamburg, Bundesstraße 55, D20146 Hamburg, Germany.

andrea.blunck@math.uni-hamburg.de

Hans Havlicek, Institut für Diskrete Mathematik und Geometrie, Technische Universität Wien, Wiedner Hauptstraße 8-10/104, A-1040 Wien, Austria.

havlicek@geometrie.tuwien.ac.at 